\title{
ON A GENERAL SYSTEM OF REPORTING AUTOPSIES IN AMERICAN ASYLUMS FOR THE INSANE.*
}

BY H. E. ALLISON, M. D.,

Superintendent of the State Asylum for Insane Criminals, Auburn, N. Y.

There are several branches of medicine, which, in their exactitude and certainty, and in the definiteness which is attached to them in all particulars, deserve to be recorded in the list of sciences. Anatomy and surgery are examples. And while it might seem presumptuous to place pathology in such a category, there are many reasons why, in this connection, it should receive some consideration. In many diseases we certainly have our knowledge so classified and arranged regarding their physical causes, their development, course and termination, that we can form a nosology as systematic and clear as any usual division made in the natural sciences. The various microscopic and diseaseproducing parasites, both animal and vegetable, that infest the body, have their natural history pretty well defined; the specific infectious diseases are quite accurately differentiated; the patho. logical changes that occur in the serous membranes as a class, or in muscular or lung tissue, of in the various organs of the body, are quite clearly understood and constant, sufficiently so to base a nomenclature of disease upon them. In a great majority of cases the expected lesion can be accurately indicated before death and found at the autopsy with a degree of positiveness that amounts to a certainty.

The foundations of medicine are laid in general pathology, especially in that division of it known as pathological anatomy or the examination of the body after death from disease. It is by an association of clinical history with pathological anatomy by means of careful corroboration upon the post-mortem table that physical diagnosis has reached its present high state of perfection. In all general diseases of the body the great majority of lesions are macroscopic in their appearances and involve such areas and such depth as to be easily distinguished by the eye. Their exact nature, however, and their finer distinctions can only be ascertained by the use of the microscope, which is an indispensable adjunct in the elucidation of the more minute disturbances, and

* Read at the annual meeting of the Association of Medical Superintendents of American Institutions for the Insane, held at Newport, R. I., June 18-20; 1889. 
particularly of those intimate changes of nutrition which constitute the initiatory steps of the process of disease. Even with its aid we cannot always discover, but in most cases can only approach a little nearer the ultimate cause. Nevertheless, the pathology of physical diseases affecting the body, including affections of the nerves and spinal cord, is largely satisfactory. The organ of the mind, alone, has been considered too subtile, too ethereal and tenuous to be grasped by the ordinary methods of anatomy and pathology, and in consequence its physical attributes, until late years, have been neglected. It is only within a very recent period that cerebral topography, or the mapping out of the various fissures and convolutions has been thought worthy of a place in our leading text books on anatomy. The fissure of Sylvius alone was recognized as permanent, the remaining surface of the brain was considered to be without definite arrangement, and was not illustrated by either plates or text. The whole surface, however, is known to conform to an exact and orderly system, and its convolutions and fissures are represented by a typical standard. The localization of function is also achieving remarkable development, especially in those areas connected with auditory, visual and motor centres.

There are very many special symptoms which are connected with local brain lesions. Various forms of paralyses are known to be produced by circumscribed cerebral degenerations, the several motor and sensory conditions found in the various forms of aphasia have special brain centres where we may look for physical causes and find them. Convulsions of special character are dependent upon localized lesions, which are demonstrable. The various cerebral tracts that pass directly to their destination and their sensory and motor connections have been traced with a degree of success that is encouraging. The localization of functions and of the paths of associated fibres have enabled diagnoses of cerebral diseases to be made with a charming degree of nicety and accuracy that warrants a certainty of prognosis and has enabled surgeons to successfully perform operations for their relief and cure. In no department but that of bacteriology has greater advance been made than in the finer anatomy of the brain, and the pathology of cerebral and spinal diseases is in line for still more satisfact ory development.

In the affections of the mind, however, there is not that positiveness of results, that we obtain in other diseases, and indeed it has been frequently stated, and in a measure the statement has 
been loosely accepted, that the brains of those dying insane do not differ notably from the brains of those sane dying outside of asylums under similar conditions as to age, environment and mode of living. This opinion has been held by many, and it should be the duty of physicians engaged in a special line of work and who alone have the opportunity, to inform the general profession what results, if any, are developed by post mortem examinations of the brain. The knowledge thus obtained should be accessible and become generally diffused. Of 235 cases examined by Dr. J. C. Howden at the Royal Lunatic Asylum, Montrose, and tabulated in the Journal of Mental Science, April, 1871, only 46 were reported as normal, and when we consider that slight but important conditions may exist and be overlooked unless the circumstances for their discovery are favorable to the examiner, some proportion of those 46 cases must undoubtedly have been evidently diseased. This paper moreover led to much discussion and thought and further investigations. Three years later Dr. W. G. Balfour, Superintendent of Hampstead Asylum, added to the number previously reported by Dr. Howden, 75 cases of Dr. Tuke's, and 390 cases collected at Colney Hatch, making 700 in all of which orily 60 , or eight and one-half per cent, were found to be normal.

We must therefore accept as a demonstrable fact that in the brains of those dying insaue we may be reasonably certain of discovering some pathological change which, in many instances, may be an adequate cause for their mental condition. These appearances, with the exception of a few instances, are not always constant in the same forms of mental disorders, at least as such disorders are at present classified. The most notable example of insanity dependent upon a determined cerebral condition is that of general paresis. This is one of the forms of mental disease that is based upon a pathological differentiation: that is, it has been selected out and made to stand apart as a distinct form of mental derangement in very much the same way as typhus fever as a separate disease was differentiated from typhoid fever thirty years ago as some members of this association will remember. Paresis occupies a place distinctively its own, and its pathology has been so determined that we may expect to find certain conditions which are definite and invariable; not always occupying the same precise areas but identically the same in its process and degenerative changes in the tissues. In this case the psychical and somatic expressions of disease indicate what morbid conditions we may uniformily expect to find. 
The condition known as idiocy has a special pathology though not altogether uniform. It may be considered as arrested or imperfect development of the cerebrum. Small crania, diminution in the cubical contents of the skull, diminished brain weights and less complex development of the fissures and convolutions are fixed conditions. While idiots are not technically insane, still they are often inmates of our asylums, and are in one sense pathological products. While a study of their brains is interesting, still they should at all times be classed as idiots, victims of a congenital defect, and as such they should be placed in a classifi. cation separate from insanity. A table of brain weights and of deficiencies and measurements in connection with them would be of value in association with embryology and brain development.

The study of psychology during the past few years has ceased to be metaphysical and abstract, but depends upon physiological and pathological conditions. It can no longer be taught apart from the physical basis of the mind, and requires for its proper understanding a knowledge of medicine and of human anatomy and physiology, particularly of the nervous centres. A pure psychosis that presents itself to us as a mental condition apart from any bodily symptom, undoubtedly exists, but only because our means of observation are imperfect and limited, for any disturb. ance of mind must depend upon some organic or functional change in the brain. This change may manifest itself in a purely psychical way unattended, so far as we can perceive, with auy bodily symptom of a physical nature-as certain forms of aphasia-and ret in these instances we can locate the cerebral lesion. There are, likewise, certain forms of mental disease in which the expression is a purely psychical one, a mental derangement without any physical disturbance. In a large majority of the insane, howcver, the disease is attended at some period of its course with active physical changes and great bodily and mental disturbance. In nearly all cases of insanity sooner or later some slight or prominent affections of the nervous system appear in their various manifestations showing that the process is a progressive and, if continued, a destructive one. The physical effects of such a morbid course are shown upon the post mortem table. Little has been done to preserve any description of the conditions there seen or to record in any public manner the pathological appearances after death in those who die insane. A few asylums have published in their annual reports some excellent notes of autopsies and have made such records a distinct and permanent part of their reports. 
It is often said that it is only the last stage of brain disease which we see in autopsies, and that the primary changes have disappeared and left nothing but the results. Even should this be so it would be well to establish a certainty as to what these results. are. Opportunities are often given us to observe the cerebral disease in the various periods of its course, as patients very commonly die of intercurrent bodily affections and not of the insanity which may then be seen in its stage of progress. In fact this is usually the ease, and all degrees of mental disorder are open for accurate description. It may not now be possible for us to generalize from such observations but some comprehensive conditions undoubtedly exist, and in the future another differentiation will be added to our list of mental diseases.

The causation of insanity which is largely speculative, its classification, the degree of education and occupation in those admitted, etc., are quite universally reported in tabular form, and it is certainly of as great importance that the appearances of the brain after death should also be made a matter of official record, as these are realities which are observed by the physician himself and do not depend upon the untrustworthy statements of others. A large mass of facts could be gathered and at the annual meetings any special subject could be designated for simultaneous investigation. Various deficiencies and improvements would suggest themselves during the course of evolution through which such $\mathbf{a}$. scheme would naturally pass.

A project of a sonewhat similar character was launched in England some years ago. It was suggested that uniform charts be used and that various aspects of the brain should be printed in diagrams upon which the lesion might be marked. Dr. Tuke, at the quarterly meeting of the Medico-Psychological Association in November, 1873, suggested that a committee he appointed to devise a uniform method of post mortem examinations. He stated that " though they might not be able to note any very great results, still as a body, by making these researches they would be preparing a storehouse of fact for other observers." Drs. J. B. Tuke, Clouston, Howilen of Montrose, Sibbald, Campbell, Howden of Waddington, and Frazer, were appointed as a committee and directed to report at a subsequent meeting. No definite action was ever taken. In August, 1888, the same subject was revived by Dr. Campbell, and Drs. Clouston, Savage, Mickle, Wigglesworth, Hack Tuke, Sibbald, Howden of Montrose, and Campbell, were appointed on the new committee. Dr. C. M. Campbell in a. 
recent letter states that the committce bas decided "that an attempt should be made to draiv up two tables, or sets of tables, the tirst of which should deal with inter-cranial appearances, the other with those of the viscera other than the brain. It was also decided that only positive appearances should be noted, and in this connection, the meeting seemed in favor of drawing a scheme for making post mortems. It was agreed that the tables should be simple, and that minute and contentious pathology should be omitted. 'The general feeling was in favor of connecting the post mortem appearances with the different forms of insanity, and, with a goorl deal of dissent, it was finally agreed to adhere to the classitication of mental disorders of the tables already existing. With regard to nomenclature, that of the latest editions of the nosology of the London College of Physicians was recommended for the sake of uniformity. On the basis of these resolutions the two sets of tables were referred to two sub-committees, each to make an attempt and submit results to the next meeting, which will take place in August."

The fact that the subject is interesting two great associations should be sufficient to denote that it is one of importance and worthy the consideration of a dignified and scientitic body of men. Some plan should be tried, not as a short-lived experiment, but adopted as a settled plan of action. Could we place in the classification of insanity but one additional form based upon pathology it would be an achievement. Insanity has too long been considered a mental cundition, apart from bodily ailments, and has received little consideration in our text books and very little in our medical schools. In fact, upon the dissection table ten years ago, the brain was sellow examined, and even now it is too infrequently used in its fresh state for the purposes of demonstration. The importance of its study is gaining ground, however, and it is safe to say that to the minute anatomy and physiology of no other organ is such attention paid as to that of the brain and spinal cord.

It is from the standpoint of physiology and pathology and not from that of metaphysics that the greatest light will ultimately be thrown upon the phenomena of insanity. This subject is one that has long attracted the attention of alienists, and various. methods have been suggested by which to accomplish the desired result. Whatever way is selected by this association, its adoption must depend npon the voluntary coöperation of its members. Various. papers have been prepared condensing the results of the post 
mortem into very brief form. Too much brevity devitalizes a report unless expressed with the utmost exactitude and in terms generally accepted as of standard technical meaning. The narrative form alone would lead to too great a diversity and lack of systematic order and arrangement. It would seem that a combination of both would best serve the purpose, and with that end in view the accompanying brief table has been prepared as a basis for general use, and to ensure an orderly, consecutive and uniform mauner of detailing results.

In the asylums of the United States there are about 50,000 patients, and approximately 3,000 deaths annually. In perbaps twelve or fifteen per cent of this number an autopsy is held, with a greater or less degree of thoroughness, and many conditions noted which, as a matter of record, should be preserved. The number of autopsies in England is much larger. Dr. James Adam* quotes from the report of the Lunacy Conmissioners in 1882 , as follows :

"We found it necessary in our last report to remark that a great falling off was to be noticed in the practice of making these very necessary examinations, and the attention of medical superintendents was drawn to the fact.

We are now glad to be able to report a great inprovement in this respect as regards returns for 1881. Of the total four thousand seven hundred and fifteen deaths which oceurred last year, two thousand seven hundred and eighty-nine were the subject of post mortem examination.

The proportion of these autopsies to the total deaths in county and borough and State asylums for the year 1880 was 37 per cent, but in 1881 it had risen to 59 per cent."

In 1884 it was still higher, i. e., 69 per cent. Very little of this material is saved. Publication is made from time to time in the journals of the occurrence of some curiosity in pathology which makes interesting reading and is thought worthy of record. It is not such cases however that are really instructive, but it is the general and universal conditions that should be preserved. We should endeavor to accumulate a large store of facts, a collection of complete totalities. From an apparently heterogeneuus mass of facts Darwin formulated the theory of the origin of species and the doctrine of evolution. Progress is a thing of slow growth and is the result of combined action of many minds. Differences of opinion undoubtedly will arise regarding what minor details are

* Path. Research in Asylums fur the Insane, Journal Mental Science, October, 1884. 
seen upon the post mortem table, but the majority of cases will be clear if we do not try to find too much and to be too elaborate. It is the common experience that the great majority of autopsies made, contain something of special interest, if ordinary care is used in making the examination, and often conditions are found that are remarkably instructive. A general record should be made in some public manner, either by publication in the annual report of each asylum, or by the appointment of some committee who should edit and publish at regular intervals such records, which should receive the sanction and endorsement of the association as a part of its official proceedings. Such publication would, in the natural course of development which attends any project, finally become more uniform, reliable and susceptible of generalization.

A careful autopsy showing negative results with a concise clinical history would be of as much relative value if thoroughly made, as one showing gross lesions. Owing to the insufficient means of examination at our disposal there would be the suspicion, in the cases of apparently normal brains, that some disease existed which was not found, still the comparative infrequency of such occurrences would show the fact that it in the minority of cases that no lesion is discovered. In the event of either plan of publication it would be necessary to depend upon the spirit of unanimity among the members of the Association. It is not expected that large results would be obtained at once, but it would certainly infuse in the minds of asylum officers a greater interest in clinical and pathological study and research, and arouse a greater thoroughness in the manner of holding autopsies. Such a course could not fail to stimulate a scientific spirit of inquiry in asylums, and to lead to an increased interest in pathology. It would be productive of a system of more careful observation of the living and would vastly enhance the importance of the patient as an individual in the study, the treatment, and in the care and cure of the insane. 